\title{
Detection of Four Lethality Types in Interspecific Crosses among Nicotiana Species through the Use of Three Rescue Methods for Lethality
}

\author{
Tetsuya Yamada, Wataru Marubashi* and Masaru Niwa
}

Laboratory of Plant Breeding, School of Agriculture, Ibaraki University, 3-21-1 Chuo, Ami-machi, Ibaraki-ken 300-0393, Japan

\begin{abstract}
Summary
Hybrid lethality was surveyed in 186 cross combinations among 17 species from 9 sections of the genus Nicotiana. The hybrid seedlings of 14 cross combinations expressed lethality characterized by one of four symptoms and the lethality was classified into four types as follows: browning of the shoot apex and the root tip (Type I), browning of the hypocotyl and the roots (Type II), yellowing of the true leaves (Type III) and formation of multiple shoots (Type IV). Three kinds of rescue methods for the lethality were applied to the 4 cross combinations expressing different types of the lethality as follows: (1) culture of hybrid seedlings under high temperature conditions to suppress the lethality, (2) culture of the cotyledonary segments of the hybrid seedlings to obtain regenerants overcoming the lethality and (3) culture of the hybrid seedlings on a medium containing cytokinin to obtain regenerants overcoming the lethality. The 1st and 2nd methods were effective in the cross of $N$. nudicaulis $\times N$. tabacum expressing the lethality Type $I$. All the methods were effective in the cross of $N$. paniculata $\times N$. suaveolens expressing the lethality Type II. Only the 1st method was effective in the cross of $N$. glutinosa $\times N$. repanda expressing the lethality Type III. None of the methods were effective in the cross of $N$. paniculata $\times N$. alata expressing the lethality Type IV. Each lethality type exhibited a specific set of responses to the three rescue methods. These findings suggest that the lethality observed in interspecific crosses of Nicotiana differs in the physiological processes leading to death.
\end{abstract}

Key Words : genus Nicotiana, cotyledon culture, cytokinin, high temperature, hybrid lethality, interspecific hybrid.

\section{Introduction}

Hybrid lethality is one of the sexual barriers preventing wide hybridization. Some cross combinations expressing the lethality have been reported in the interspecific hybrids of several genera, such as Triticum (Chen et al. 1989), Oryza (Oka 1962) and Gossypium (Philips 1977).

Communicated by T. Adachi

Received March 23, 1999. Revision received June 25, 1999.

Accepted July 12, 1999.

*Corresponding author
In the genus Nicotiana, Kostoff (1930) performed the hybridization of 80 cross combinations among 16 species, and observed that the hybrids of 9 cross combinations died at the seedling stage. De Verna et al. (1987) performed 10 reciprocal crosses between $N$. tabacum cv. 'Ky 17 ' and 5 wild species in Nicotiana using in vitro pollination and ovule culture, and reported that the seedlings derived from 2 crosses always displayed lethal necrosis. However, the lethal symptoms displayed by these hybrid seedlings were not described in detail.

It is important to overcome the lethality in interspecific hybrids to widen genetic variation in cultivated species. So far, rescue methods for the lethality have been developed in a few cross combinations of the Nicotiana species. For example, in the cross of $N$. suaveolens $\times N$. tabacum, three rescue methods have been developed. Marubashi et al. (1988) reported that the lethality was suppressed by culturing the hybrid seedlings under high temperature conditions $\left(36^{\circ} \mathrm{C}\right)$. Lloyd (1975) cultured cotyledonary segments of the hybrid seedlings on media containing plant growth regulators and obtained viable hybrids from regenerants through the callus. Inoue et al. $(1994,1997)$ cultured the intact hybrid seedlings on media containing cytokinins and obtained viable hybrids derived from adventitious buds that were regenerated from the hypocotyl of dying hybrid plants. Through the use of these three rescue methods, it was anticipated that the physiological processes involved in the lethality could be elucidated along with the primary objective of rescuing the hybrids from lethality.

In this study, we surveyed the cross combinations expressing lethality among the Nicotiana species and classified the lethality based on the symptoms. Furthermore, we applied three rescue methods to the hybrid seedlings showing different lethal symptoms and attempted to analyze the physiological characteristics of lethality in interspecific crosses of the genus Nicotiana.

\section{Materials and Methods}

\section{Plant materials}

Seventeen species of Nicotiana were used in this experiment (Table 1). The seeds of these species were supplied by Japan Tobacco Inc. and maintained in our laboratory. Plants were grown in a greenhouse of the School of Agriculture, Ibaraki University from February to December in 1994. 
Table 1. Nicotiana species used in the interspecific hybridization

\begin{tabular}{llc}
\hline Section ${ }^{1}$ & Species & $\begin{array}{c}\text { Haploid } \\
\text { chromosome } \\
\text { numbers }\end{array}$ \\
\hline Paniculatae & N. glauca & 12 \\
Rusticae & N. paniculata & 12 \\
Tomentosae & N. rustica & 24 \\
Genuinae & N. glutinosa & 12 \\
Trigonophyrllae & N. otophora & 12 \\
Alatae & N. tabacum & 24 \\
& N. trigonophylla & 12 \\
& N. alata & 9 \\
Repandae & N. plumbaginifolia & 10 \\
Nudicaules & N. sylvestris & 10 \\
Suaveolentes & N. repanda & 12 \\
& N. nudicaulis & 24 \\
N. suaveolens & 24 \\
N. gossei & 16 \\
N. megalosiphon & 19 \\
& N. debney & 20 \\
& & 24 \\
\hline
\end{tabular}

1) According to the description of Goodspeed (1954).

\section{Cross-pollination}

Cross-pollination was performed reciprocally between two species whose flowering time was compatible. Flowers of the maternal species, which were emasculated before flowering, were pollinated with fresh pollen of the paternal species. Thus, 186 cross-pollinations were successful among 17 species.

\section{Germination of hybrid seeds}

Ten $F_{1}$ seeds for each successful cross were soaked in a $0.5 \%$ Gibberellin $\mathrm{A}_{3}$ solution for 30 minutes, sterilized with a $5 \%$ sodium hypochlorite solution $(0.25 \%$ liberated chloride) for 15 minutes and rinsed three times with sterilized water. $F_{1}$ seeds were sown on MS medium (Murashige and Skoog 1962), with half the concentration of salts, supplemented with $1 \%$ sucrose and $0.2 \%$ gelrite ( $\mathrm{pH}$ 5.8), and were cultured at $28{ }^{\circ} \mathrm{C}$ under continuous illumination $(\mathrm{ca} .3,000 \mathrm{~lx})$ for germination. Under these conditions, lethality was expressed in the crosses of $N$. suaveolens $\times N$. tabacum (Manabe et al. 1989). Lethal symptoms were investigated in the hybrids throughout the growth period. Surviving seedlings were transferred from the medium to soil at 60 days after germination.

Culture under high temperature conditions to suppress the lethality-high temperature culture

Germinating hybrids were exposed to six different temperatures $\left(28,30,32,34,36,38{ }^{\circ} \mathrm{C}\right)$ under continuous illumination $(\mathrm{ca} .3,000 \mathrm{~lx})$. All the seedlings were observed for the expression of lethality after the initiation of high temperature culture. At 45 days after germination, the surviving seedlings were transferred from the medium to soil in pots. Half of them grew at the same temperature and the others were exposed to the lethal temperature of $28^{\circ} \mathrm{C}$ to confirm the suppression of the lethality by the high temperature conditions (Manabe et al. 1989).

Culture of cotyledonary segments to overcome the lethality-cotyledon culture

Cotyledon culture was performed according to the method of Lloyd (1975). A cotyledon of the hybrid seedling was cut into four transverse segments $2-3 \mathrm{~mm}$ in width. The segments were placed on MS medium, supplemented with $2 \mathrm{mg} l^{-1}$ of $\alpha$-naphthalenacetic acid (NAA), $2 \mathrm{mg} \mathrm{l}^{-1}$ of 6 -benzylaminopurine (BAP), 3\% sucrose and $0.8 \%$ agar ( $\mathrm{pH} 5.5)$, and cultured at $28{ }^{\circ} \mathrm{C}$ under continuous illumination (ca. 3,000 1x) for dedifferentiation and shoot formation. For rooting, the regenerated shoots were transferred to MS medium, supplemented with $2 \mathrm{mg} l^{-1} \mathrm{NAA}, 3 \%$ sucrose and $0.8 \%$ agar ( $\mathrm{pH} 5.5)$.

Culture of hybrid seedlings on the medium containing cytokinin to overcome the lethality-cytokinin culture Cytokinin culture was performed according to the method of Inoue et al. (1994). The hybrid seedlings were transferred to MS medium, with half the concentration of salts, supplemented with $0-10 \mathrm{mg} l^{-1}$ BAP,

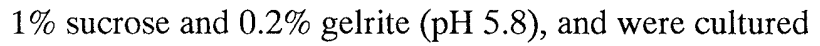
at $28^{\circ} \mathrm{C}$ under continuous illumination $(\mathrm{ca} .3,000 \mathrm{~lx}$ ). For rooting, the adventitious shoots formed on the seedlings were cut and transferred to MS medium, with half the concentration of salts, supplemented with $2 \mathrm{mg} l^{-1}$ NAA, $1 \%$ sucrose and $0.2 \%$ gelrite ( $\mathrm{pH} 5.8$ ).

\section{Chromosome observation}

In the above two methods for overcoming lethality, all the surviving regenerants were transferred from the medium to soil and were grown to maturity in a greenhouse. Chromosomes were stained with a basic fuchsin solution and were counted under a light microscope.

\section{Results}

\section{Survey of lethality}

Pod formation was observed in 108 of the 186 cross combinations performed. Germination was observed in 61 cross combinations. The hybrid seedlings from 14 cross combinations expressed the lethality and died within 60 days after germination, whereas, no lethal symptoms were observed in the other 47 cross combinations.

Four lethality types were detected in the above 14 cross combinations based on the lethal symptoms of the seedlings by which the cross combinations were clas- 
Table 2. Lethality types detected in the interspecific crosses of Nicotiana

\begin{tabular}{|c|c|c|}
\hline Lethality type & Lethal symptoms ${ }^{1)}$ & Cross combinations $^{2)}$ \\
\hline Type I & Browning of shoot apex and root tip & $N$. nudicaulis $\times N$. tabacum \\
\hline Type II & Browning of hypocotyl and roots & $\begin{array}{l}N . \text { paniculata } \times N . \text { gossei } \\
N . \text { paniculata } \times N \text {. suaveolens } \\
N . \text { debneyi } \times N \text {. tabacum } \\
N . \text { debneyi } \times N \text {. sylvestris } \\
N \text {. debneyi } \times N \text {. repanda } \\
\end{array}$ \\
\hline Type III & Yellowing of true leaves & $\begin{array}{l}N . \text { paniculata } \times N . \text { nudicaulis } \\
N . \text { glutinosa } \times N . \text { repanda } \\
N . \text { trigonophylla } \times N \text {. otophora } \\
N . \text { megalosiphon } \times N \text {. longiflora } \\
N . \text { megalosiphon } \times N \text {. plumbaginifolia }\end{array}$ \\
\hline Type IV & Formation of multiple shoots & $\begin{array}{l}N \text {. paniculata } \times N \text {. glutinosa } \\
N . \text { paniculata } \times N \text {. alata } \\
N \text {. gossei } \times N \text {. alata }\end{array}$ \\
\hline
\end{tabular}

1) All the symptoms were observed in the hybrid seedlings by 45 days after germination.

${ }^{2)}$ All the seedlings which germinated showed the lethal symptoms.
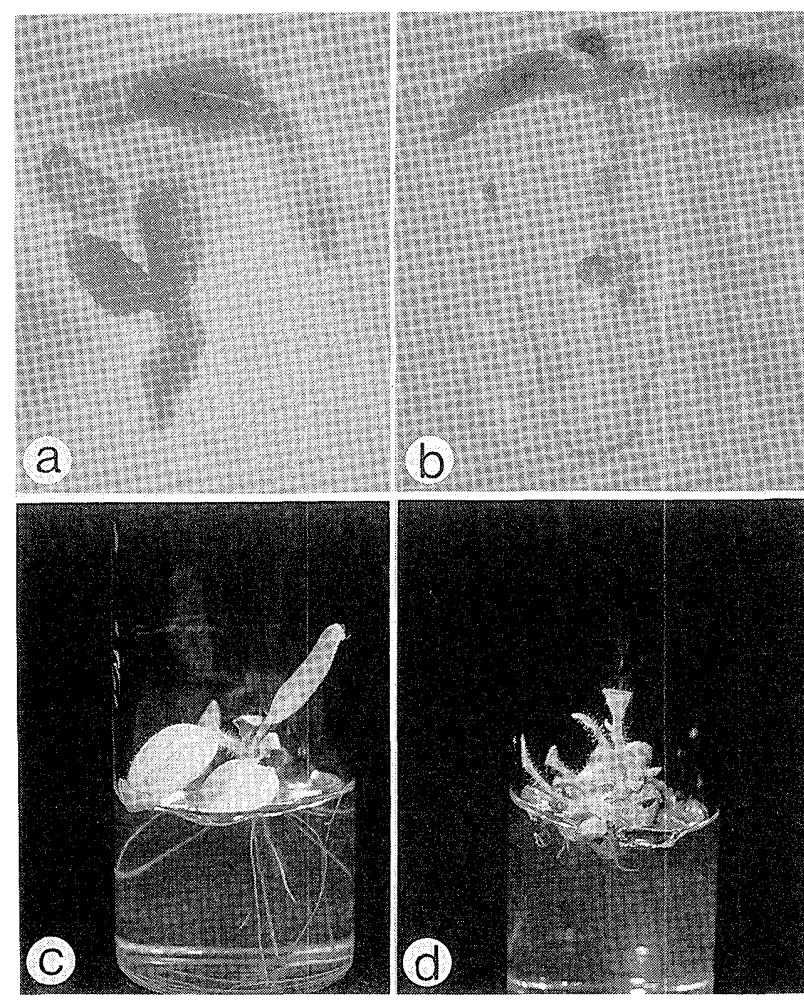

Fig. 1. Lethal symptoms observed in the hybrid seedlings of the Nicotiana cross combinations: a, browning of shoot apex and root tip at the time of germination in the cross $N$. nudicaulis $\times N$. tabacum; $\mathrm{b}$, browning of hypocotyl and roots one week after germination in the cross $N$. paniculata $\times N$. suaveolens; c, yellowing of true leaves at 3 weeks after germination in the cross $N$. glutinosa $\times N$. repanda; $\mathrm{d}$, formation of multiple shoots at 3 weeks after germination in the cross $N$. paniculata $\times N$. alata. sified (Table 2). In the cross of $N$. nudicaulis $\times N$. tabacum, the hybrid seedlings showed browning of the shoot apex and the root tip (Fig. 1a). Lethality characterized by these symptoms was not detected in any of the other combinations and was referred to as Type I. In the cross of $N$. paniculata $\times N$. suaveolens, the hybrid seedlings showed browning of the hypocotyl and the roots (Fig. 1b). Lethality characterized by these symptoms was detected in the 5 combinations and referred to as Type II. In the cross of $N$. glutinos $a \times N$. repanda, the hybrid seedlings showed yellowing of the true leaves (Fig. 1c). Lethality associated with this symptom was detected in the 5 combinations and referred to as Type III. In the cross of $N$. paniculata $\times N$. alata, the multiple shoots were formed on the basal part of the seedlings (Fig. 1d) and underwent browning. Lethality characterized by this symptom was detected in the three combinations and referred to as Type IV.

In order to analyze the physiological characteristics of the lethality, 3 rescue methods were applied to 1 cross combination from each lethality type; $i$. e. N. nudicaulis $\times N$. tabacum (Type I), N. paniculata $\times N$. suaveolens (Type II), $N$. glutinosa $\times N$. repanda (Type III) and $N$. paniculata $\times N$. alata (Type IV). These cross combinations were selected for their high seed setting and high germination rate.

\section{Effect of high temperature culture}

The hybrid seedlings of the above 4 cross combinations showed different responses under the various temperature conditions (Table 3). Hybrid seedlings of $N$. nudicaulis $\times N$. tabacum (Type I) and N. paniculata $\times$ $N$. suaveolens (Type II) expressed the lethality at 28 and $30{ }^{\circ} \mathrm{C}$ but not at $32-38^{\circ} \mathrm{C}$ (Fig. 2a, b). Hybrid seedlings of $N$. glutinosa $\times N$. repanda (Type III) died within 30 
Table 3. Effect of temperature conditions on the growth of hybrid seedlings derived from four Nicotiana crosses

\begin{tabular}{|c|c|c|c|c|}
\hline \multirow{2}{*}{ Cross combination } & \multirow{2}{*}{$\begin{array}{c}\text { Culture } \\
\text { temperature } \\
\left({ }^{\circ} \mathrm{C}\right)\end{array}$} & \multirow{2}{*}{$\begin{array}{l}\text { No. of } F_{1} \\
\text { seedlings } \\
\text { cultured }\end{array}$} & \multicolumn{2}{|c|}{ No. of surviving seedlings } \\
\hline & & & $30^{1)}$ & 45 \\
\hline \multirow[t]{6}{*}{ N. nudicaulis $\times$ N. tabacum } & 28 & 10 & 0 & 0 \\
\hline & 30 & 10 & 0 & 0 \\
\hline & 32 & 16 & 16 & 16 \\
\hline & 34 & 18 & 18 & 18 \\
\hline & 36 & 10 & 10 & 10 \\
\hline & 38 & 15 & 15 & 15 \\
\hline \multirow[t]{6}{*}{ N. paniculata $\times N$. suaveolens } & 28 & 16 & 0 & 0 \\
\hline & 30 & 14 & 0 & 0 \\
\hline & 32 & 14 & 14 & 14 \\
\hline & 34 & 16 & 16 & 16 \\
\hline & 36 & 15 & 15 & 15 \\
\hline & 38 & 16 & 16 & 16 \\
\hline \multirow[t]{6}{*}{$N$. glutinosa $\times N$. repanda } & 28 & 10 & 0 & 0 \\
\hline & 30 & 10 & 0 & 0 \\
\hline & 32 & 10 & 6 & 6 \\
\hline & 34 & 10 & 0 & 0 \\
\hline & 36 & 10 & 0 & 0 \\
\hline & 38 & 10 & 0 & 0 \\
\hline \multirow[t]{6}{*}{$N$. paniculata $\times N$. alata } & 28 & 10 & 4 & 0 \\
\hline & 30 & 10 & 6 & 0 \\
\hline & 32 & 10 & 10 & 0 \\
\hline & 34 & 10 & 6 & 0 \\
\hline & 36 & 10 & 7 & 0 \\
\hline & 38 & 10 & 0 & 0 \\
\hline
\end{tabular}

1) Days after germination.

days at $28-30{ }^{\circ} \mathrm{C}$ and showed chlorosis and stopped growing after 30 days at $34-38{ }^{\circ} \mathrm{C}$ (Fig. 2c). In this combination at $32{ }^{\circ} \mathrm{C}$, hybrid seedlings showed a delay in the appearance of the lethal symptoms and survived for 45 days after germination. In the cross of $N$. paniculata $\times N$. alata (Type IV), some of the 10 hybrid seedlings expressed the lethality within 30 days after germination at all the temperatures except for $32{ }^{\circ} \mathrm{C}$. All the hybrid seedlings, including hybrid plants which did not display lethal symptoms at first, showed necrosis and died within 45 days after germination.

Hybrid seedlings of $N$. nudicaulis $\times N$. tabacum and $N$. paniculata $\times N$. suaveolens grew to maturity under the high temperature conditions $\left(32-38^{\circ} \mathrm{C}\right)$. When exposed to $28^{\circ} \mathrm{C}$, they died after showing the typical symptoms.

\section{Effect of cotyledon culture}

In the 4 cross combinations, the cotyledonary segments behaved differently on the medium for dedifferentiation and shoot formation (Table 4). In the cross of $N$. nudicaulis $\times N$. tabacum, all the segments showed browning and no further growth. In the cross of $N$. paniculata $\times N$. suaveolens, 15 of the 27 segments formed calli and many vigorous shoots were regenerated from the surface of the calli (Fig. 3a, b). In the cross of $N$. glutinosa $\times N$. repanda, 3 of the 25 segments formed calli and some shoots were regenerated. These shoots showed chlorosis and did not grow further. In the cross of $N$. paniculata $\times N$. alata, 8 of the 10 segments formed calli. These calli showed browning and failed to grow.

In the cross of $N$. paniculata $\times N$. suaveolens, vigorous shoots regenerated roots on the rooting medium (Fig. $3 c)$. The regenerants showed vigorous growth and became mature (Fig. 3d). The chromosome number of the somatic cells was 28 , which corresponded to the combined number of the parental species. Cotyledon culture was effective in overcoming the lethality in this cross combination.

\section{Effect of cytokinin culture}

When cultured on the media containing cytokinin (BAP) at various concentrations, the hybrid seedlings derived from the 4 cross combinations showed different reactions after expressing the lethality (Table 5). In the cross of $N$. nudicaulis $\times N$. tabacum, the seedlings showing 

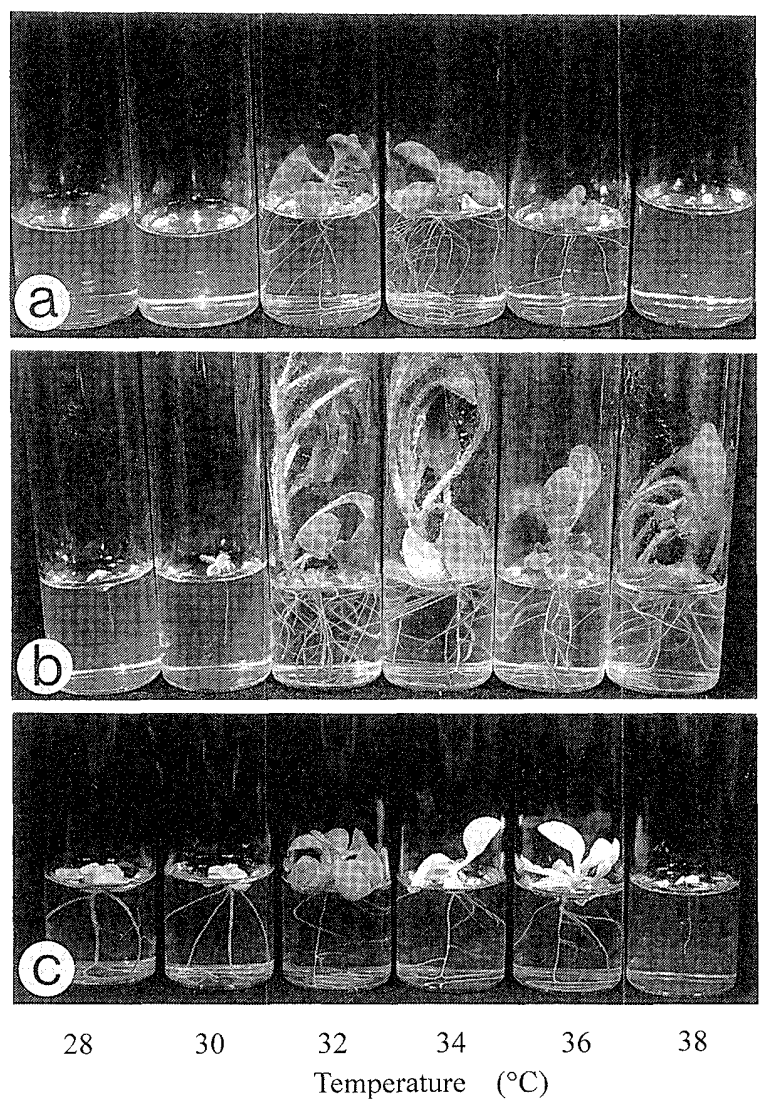

Fig. 2. Hybrid seedlings cultured at various temperatures at 30 days after germination: a, cross $N$. nudicaulis $\times N$. tabacum; b, cross $N$. paniculata $\times N$. suaveolens; c, cross $N$. glutinosa $\times N$. repanda.

Table 4. Growth of callus and shoots from the cotyledonary segments of hybrid seedlings derived from four Nicotiana crosses

\begin{tabular}{|c|c|c|c|c|}
\hline \multirow{2}{*}{ Cross combination } & \multirow{2}{*}{$\begin{array}{c}\text { No. of } \\
\text { segments } \\
\text { cultured }\end{array}$} & \multicolumn{3}{|c|}{ No. of segments with ${ }^{1)}$} \\
\hline & & $\begin{array}{l}\text { only } \\
\text { callus }\end{array}$ & $\begin{array}{l}\text { callust } \\
\text { shoots }\end{array}$ & $\begin{array}{c}\text { no } \\
\text { response }\end{array}$ \\
\hline N. nudicaulis $\times N$. tabacum & 25 & 0 & 0 & 25 \\
\hline N. paniculata $\times N$. suaveolens & 27 & 0 & 15 & 12 \\
\hline$N$. glutinosa $\times N$. repanda & 25 & 0 & 3 & 22 \\
\hline$N$. paniculata $\times N$. alata & 10 & 8 & 0 & 2 \\
\hline
\end{tabular}

1) The data were obtained 30 days after the initiation of culture.

symptoms of the lethality formed calli from the browning hypocotyl on the media containing 0.2 or $2 \mathrm{mg} \mathrm{l}^{-1}$ BAP (Fig. 4a). On the medium containing $0.2 \mathrm{mg} l^{-1}$ $\mathrm{BAP}$, the calli regenerated numerous vigorous shoots. In the cross of $N$. paniculata $\times N$. suaveolens, the seedlings showing symptoms of the lethality regenerated numerous vigorous shoots directly from the browning hypocotyl on the media containing $0.2-10 \mathrm{mg} l^{-1}$ BAP (Fig. $4 \mathrm{~b})$. In the cross of $N$. glutinosa $\times N$. repanda, the seedlings showing symptoms of the lethality formed calli from the root tip on the media containing 2 or 10
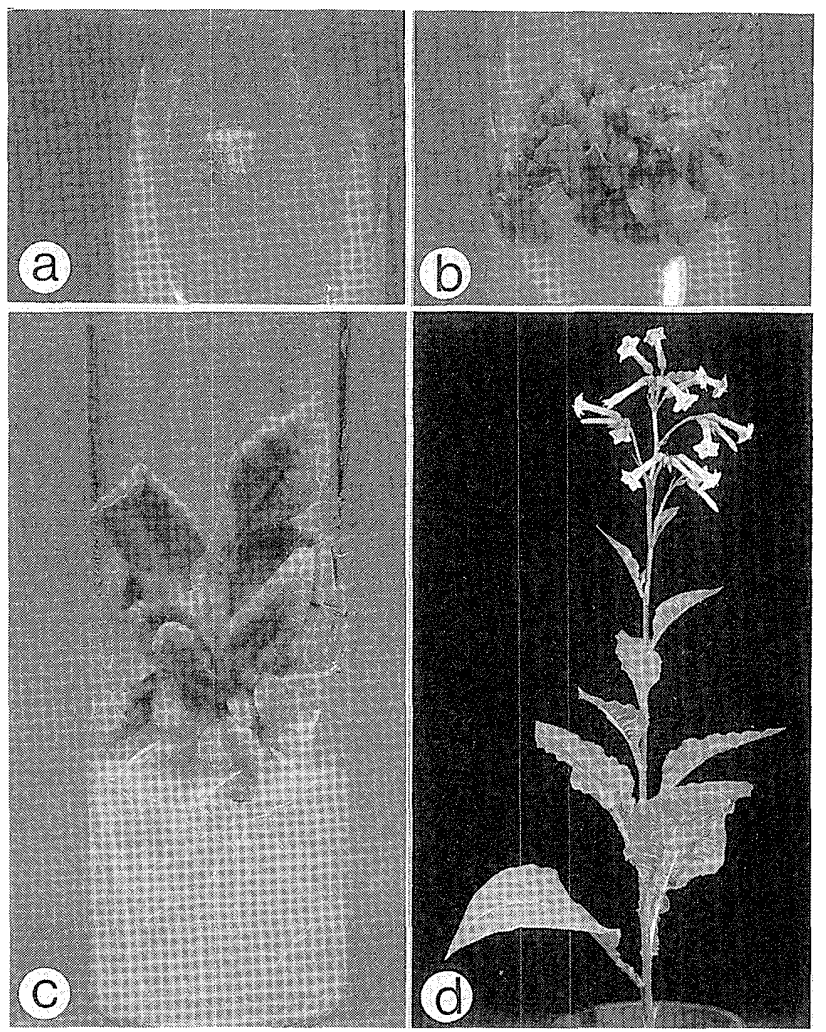

Fig. 3. Raising process of surviving hybrid plants through culture of the cotyledonary segment of a hybrid seedling in the cross $N$. nudicaulis $\times N$. tabacum: a, callus formed from a cotyledonary segment at 7 days after initiation of the culture; $b$, multiple shoots redifferentiated from the callus at 30 days after initiation of the culture; $c$, vigorous plant regenerated from the shoot at 60 days after initiation of the culture; $d$, mature plant at 4 month after initiation of the culture.

$\mathrm{mg} l^{-1}$ BAP. On the medium containing $10 \mathrm{mg} l^{-1}$ BAP, the calli regenerated shoots. These shoots showedchlorosis and did not grow further. In the cross of $N$. paniculata $\times N$. alata, the seedlings exhibiting chlorosis formed calli from the hypocotyl on the media containing $0.2-10 \mathrm{mg}^{-1}$ BAP. All the calli showed browning and did not grow further.

The vigorous shoots of $N$. nudicaulis $\times N$. tabacum, $N$. glutinosa $\times N$. repanda regenerated roots on the medium for rooting. These regenerants displayed vigorous growth and matured. The chromosome numbers of the somatic cells were 28 and 48 respectively, which corresponded to the combined number of the parental species. Culture on the media containing cytokinin was effective in overcoming the lethality in these 2 cross combinations.

\section{Classification of lethality}

Three rescue methods showed different effects on the four lethality types (Table 6). The lethality types were classified according to the responses of the hybrid seedlings to the rescue methods. In the case of lethality Type I, high temperature conditions and cytokinin culture exerted beneficial effects unlike cotyledon cul- 
Table 5. Effect of cytokinin on callus or shoot formation in hybrid seedlings derived from four Nicotiana crosses

\begin{tabular}{|c|c|c|c|c|c|c|}
\hline \multirow[b]{2}{*}{ Cross combination } & \multirow{2}{*}{$\begin{array}{c}\text { BAP }^{1)} \\
\text { concentration } \\
(\mathrm{m} / l)\end{array}$} & \multirow{2}{*}{$\begin{array}{l}\text { No. of } \\
\text { seedlings } \\
\text { cultured }\end{array}$} & \multicolumn{4}{|c|}{ No. of seedlings with ${ }^{2)}$} \\
\hline & & & $\begin{array}{l}\text { only } \\
\text { callus }\end{array}$ & $\begin{array}{l}\text { callust } \\
\text { shoots }\end{array}$ & $\begin{array}{l}\text { only } \\
\text { shoots }\end{array}$ & $\begin{array}{c}\text { no } \\
\text { response }\end{array}$ \\
\hline \multirow[t]{5}{*}{$N$. nudicaulis $\times N$. tabacum } & 0 & 8 & 0 & 0 & 0 & 8 \\
\hline & 0.01 & 9 & 0 & 0 & 0 & 9 \\
\hline & 0.2 & 11 & 0 & 11 & 0 & 0 \\
\hline & 2 & 10 & 7 & 0 & 0 & 3 \\
\hline & 10 & 6 & 0 & 0 & 0 & 6 \\
\hline \multirow[t]{5}{*}{ N. paniculata $\times N$. suaveolens } & 0 & 8 & 0 & 0 & 0 & 8 \\
\hline & 0.01 & 8 & 0 & 0 & 0 & 8 \\
\hline & 0.2 & 9 & 0 & 0 & 8 & 1 \\
\hline & 2 & 6 & 0 & 0 & 5 & 1 \\
\hline & 10 & 6 & 0 & 0 & 4 & 2 \\
\hline \multirow[t]{5}{*}{$N$. glutinosa $\times N$. repanda } & 0 & 15 & 0 & 0 & 0 & 15 \\
\hline & 0.01 & 15 & 0 & 0 & 0 & 15 \\
\hline & 0.2 & 15 & 0 & 0 & 0 & 15 \\
\hline & 2 & 15 & 15 & 0 & 0 & 0 \\
\hline & 10 & 11 & 9 & 2 & 0 & 0 \\
\hline \multirow[t]{5}{*}{$N$. paniculata $\times N$. alata } & 0 & 4 & 0 & 0 & 0 & 4 \\
\hline & 0.01 & 5 & 0 & 0 & 0 & 5 \\
\hline & 0.2 & 3 & 2 & 0 & 0 & 1 \\
\hline & 2 & 4 & 1 & 0 & 0 & 3 \\
\hline & 10 & 1 & 1 & 0 & 0 & 0 \\
\hline
\end{tabular}

1) 6-benzylaminopurine.

2) The data were obtained 60 days after the initiation of culture.
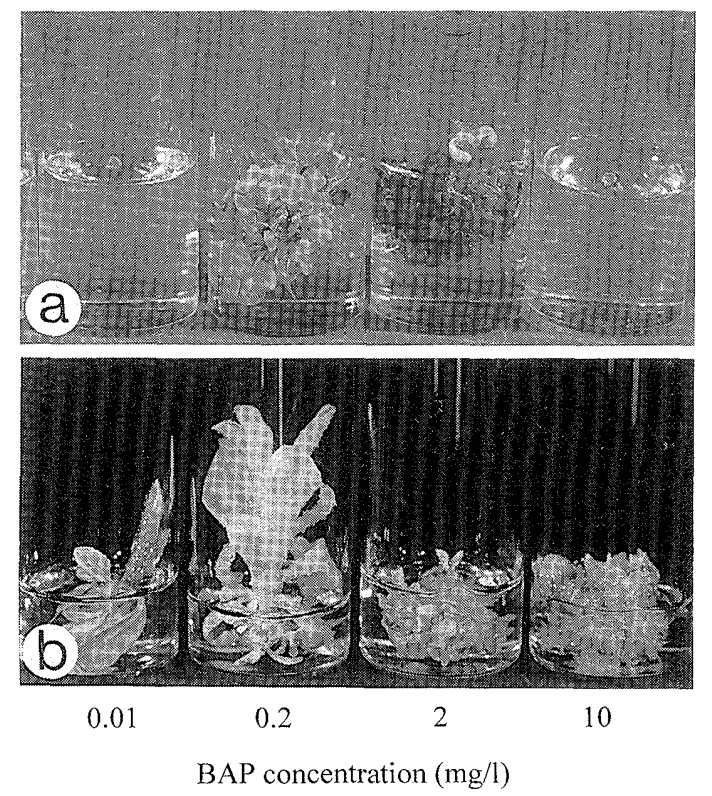

Fig. 4. Formation of vigorous shoots and calli through the culture of hybrid seedlings on the media containing cytokinin (BAP) of 60 days after initiation of the culture: a, cross $N$. nudicaulis $\times N$. tabacum; $\mathrm{b}$, cross $N$. paniculata $\times N$. suaveolens. ture. All the methods exerted beneficial effects in the case of lethality Type II. In the case of lethality Type III, high temperature conditions suppressed the lethality but other methods did not have a beneficial effect. None the methods were effective in the case of lethality Type IV. The set of responses to the three rescue methods was specific to the four lethality types.

\section{Discussion}

Hybrid lethality is one of the constraints to be overcome in plant breeding. In this study, we characterized the lethality expressed in Nicotiana crosses. Fourteen cross combinations expressing the lethality were detected in 186 cross combinations of the Nicotiana species from 9 sections. The cross combinations were classified into four types based on the lethal symptoms. Through the use of three rescue methods, different effects were observed in each lethality type. Moreover, each lethality type showed a specific set of responses to the three rescue methods.

The lethality that had been previously reported in detail in the Nicotiana crosses corresponded to the four types observed in this study. From the description of the lethal symptoms in the previous studies, lethality expressed in the 2 crosses of $N$. suaveolens $\times N$. tabacum 
Table 6. Response to three rescue methods in hybrid seedlings

\begin{tabular}{|c|c|c|c|c|}
\hline \multirow[t]{2}{*}{ Cross combination } & \multirow{2}{*}{$\begin{array}{l}\text { Lethality } \\
\text { type }\end{array}$} & \multicolumn{3}{|c|}{ Response to } \\
\hline & & high temperature & cotyledon culture & cytokinin culture \\
\hline$N$. nudicaulis $\times N$. tabacum & I & suppressing ${ }^{1)}$ & no effect $^{2)}$ & overcoming ${ }^{3)}$ \\
\hline$N$. paniculata $\times N$. suaveolens & II & suppressing & overcoming & overcoming \\
\hline$N$. glutinosa $\times N$. repanda & III & suppressing & no effect & no effect \\
\hline$N$. paniculata $\times N$. alata & IV & no effect & no effect & no effect \\
\hline
\end{tabular}

${ }^{1)}$ Seedlings had been kept continuously under high temperature conditions.

${ }^{2)}$ No viable seedlings or regenerated plants were obtained.

${ }^{3)}$ Regenerated plants were obtained.

(Lloyd 1975, Marubashi et al. 1988) and N. suaveolens $\times N$. sylvestris (Inoue et al. 1996) was identified as Type II. Similarly, lethality expressed in the cross of $N$. repanda $\times N$. tabacum (Iwai et al. 1984) was identified as Type III. Type II has been detected in 8 cross combinations and Type III in 6 cross combinations including the findings in a previous study.

Type I and IV had not been reported and were detected in a few cross combinations (Type I; 1 cross combination, Type IV; 3 cross combinations) in our survey. Type I was expressed between Genuinae and Nudicaules at the level of sections (Table 1). These two sections include only one species respectively; $i$. e. $N$. tabacum and $N$. nudicaulis (Goodspeed 1954). This type may be restricted to the combination of these two sections. In Type IV, most of the seedlings formed multiple shoots and underwent gradual browning, together with the shoots. However, a few seedlings were able to grow without multiple shoots, but they also underwent browning and died within 40 days after germination. Thus, we detected new types of lethality expressed in the Nicotiana crosses.

According to the previous studies in 2 cross combinations $(N$. suaveolens $\times N$. tabacum, $N$. repanda $\times N$. tabacum) expressing the lethality, the responses to the rescue methods of the seedlings were in agreement with our results for the same lethality type shown in Table 6, respectively. In the cross of $N$. suaveolens $\times N$. tabacum expressing the lethality Type II, high temperature conditions (Manabe et al. 1989), cotyledon culture (Lloyd 1975) and cytokinin culture (Inoue et al. 1994) exerted beneficial effects in the hybrid. In the cross of $N$. repanda $\times N$. tabacum expressing lethality Type III, high temperature conditions had beneficial effects (Suda and Marubashi 1990), unlike cotyledon culture (Iwai et al. 1984) in the hybrid. Therefore, it is suggested that the responses to the rescue methods are specific to each lethality type.

In this study, we carried out morphological and physiological characterization of the lethality and detected four lethality types in the interspecific crosses of Nicotiana. It is suggested that the lethality observed in the Nicotiana crosses undergoes different physiological processes leading to death.

\section{Acknowledgments}

We thank Dr. Eiichi Inoue, School of Agriculture, University of Ibaraki, for his technical advice. This work was partly supported by the Sasakawa Scientific Research Grant from The Japan Science Society.

\section{Literature Cited}

Chen, N. C., D. A. Evans and A. Vasconcelos (1989) Use of tissue culture to bypass wheat hybrid necrosis. Theor. Appl. Genet. 78: 57-60.

De Verna, J. W., J. R. Mayers and G. B. Collins (1987) Bypassing prefertilization barriers to hybridization in Nicotiana using in vitro pollination and fertilization. Theor. Appl. Genet. 73:665-671.

Goodspeed, T. H. (1954) The genus Nicotiana. Chronica Botanica, Waltham Mass.

Inoue, E., W. Marubashi and M. Niwa (1994) Simple method for overcoming the lethality observed in the hybrid between Nicotiana suaveolens and N. tabacum. Breed. Sci. $44: 333-336$.

—_ - and - (1997) Improvemant of the method for overcoming the hybrid lethality between Nicotiana suaveolens and $N$. tabacum by culture of $F_{1}$ seeds in liquid media containing cytokinins. Breed. Sci. 47:211-216.

Iwai, S., C. Kishi, K. Nakata, N. Kawashima and S. Kubo (1984) Production of interspecific hybrids by ovule culture. Jpn. J. Breed. 34[Suppl 1]:60-61.

Kostoff, D. (1930) Ontogeny, genetics and cytology of Nicotiana hybrids. Genetica 12:33-139.

Lloyd, R. (1975) Tissue culture as means of circumventing lethality in an interspecific Nicotiana hybrid. Tob. Sci. $19: 4-6$.

Manabe, T., W. Marubashi and Y. Onozawa (1989) Temperature-dependent conditional lethality in interspecific hybrids between Nicotiana suaveolens Lehm. and $N$. tabacum L. Proc. of the 6th Internatl. Congr. of SABRAO, pp. $459-462$.

Marubashi, W., I. Tatsuno and Y. Onozawa (1988) Effect of high temperature on the growth of hybrid lethal seedlings 
between Nicotiana suaveolens Lehm. $\times N$. tabacum L. Jpn. J. Breed. 38 [Suppl 1]:66-67.

Murashige, T. and F. Skoog (1962) A revised medium for rapid growth and bio assays with tobacco tissue culture. Physiol. Plant. 15:473-497.

Oka, H. (1962) Phylogenetic differentiation of cultivated rice, $\mathrm{XX}$. Analysis of the genetic basis of hybrid breakdown in rice. Japan J. Genet. 37(1): 24-35.
Philips, L. L. (1977) Interspecific incompatibility in Gossypium. IV. Temperature-conditional lethality in hybrids of G. klotzschianum. Amer. J. Bot. 64(7):914-915.

Suda, M. and W. Marubashi (1990) Effects of culture temperatures and IAA on the growth of hybrid seedlings of Nicotiana repanda Willd. $\times$ N. tabacum L. Jpn. J. Breed. 40[Suppl 1]:58-59. 